\title{
A diffusive description of the focused transport of solar energetic particles
}

\section{Intensity- and anisotropy-time profiles as a powerful diagnostic tool for interplanetary particle transport conditions ${ }^{\star}$}

\author{
S. Artmann ${ }^{1}$, R. Schlickeiser ${ }^{1}$, N. Agueda ${ }^{2,3}$, S. Krucker ${ }^{2}$, and R. P. Lin ${ }^{2,4,5}$ \\ ${ }^{1}$ Institut für Theoretische Physik, Lehrstuhl IV: Weltraum- und Astrophysik, Ruhr-Universität Bochum, 44780 Bochum, Germany \\ e-mail: sa@tp4.rub.de \\ 2 Space Sciences Laboratory, University of California, Berkeley, CA 94720-7450, USA \\ 3 Departament d'Astronomia i Meteorologia, Institut de Ciències del Cosmos (ICC), Universitat de Barcelona, Spain \\ 4 Physics Department, University of California, Berkeley, CA 94720-7300, USA \\ 5 School of Space Research, Kyung Hee University, Yongin, Gyeonggi, Korea
}

Received 15 August 2011 / Accepted 9 September 2011

\begin{abstract}
The transport of solar energetic charged particles along the interplanetary magnetic field in the ecliptic plane of the sun can be described roughly by a one-dimensional diffusion equation. Large-scale spatial variations of the guide magnetic field can be taken into account by adding an additional term to the diffusion equation that includes the effect of adiabatic focusing. We solve this equation analytically by assuming a point-like particle injection in time and space and a spatial power-law dependence for the focusing length and the spatial diffusion coefficient. We infer the intensity- and anisotropy-time profiles of solar energetic particles from this solution. Through these the influence of different assumptions for the diffusion parameters can be seen in a mathematically closed form. The comparison of calculated and measured intensity- and anisotropy-time profiles, which are a powerful diagnostic tool for interplanetary particle transport, gives information about the large-scale spatial dependence of the focusing length and the diffusion coefficient. For an exceptionally large solar energetic particle event, which did occur on 2001 April 15, we fit the 27-512 keV electron intensities and anisotropies observed by the Wind spacecraft using the theoretically derived profiles. We find a linear spatial dependence of the mean free path along the guiding magnetic field. We also find the mean free path to be energy independent, which supports the theory of "velocity-dependent diffusion". This means that the intensity profiles for the discussed energies exhibit the same shape if they are plotted against the traveled distance and not against the time. In this case the profiles differ only in their maximum values and we can determine the energy spectra of the solar flare electrons out of the scaling factor we need to fit the data. The derived spectra exhibits a power-law dependence $\propto E_{\text {kin }}^{-3}$ in an energy range from $\sim 50 \mathrm{keV}$ to $\sim 500 \mathrm{keV}$.
\end{abstract}

Key words. solar wind - Sun: magnetic topology - scattering - diffusion - stars: flare

\section{Introduction}

Modeling the transport of solar energetic particles has been in the focus of interest for several years. Most models assume a large-scale structure of a guide magnetic field, $\boldsymbol{B}_{0}$, with superposed small irregularities, $\delta \boldsymbol{B}$. The energetic charged particles move along this guide magnetic field $\boldsymbol{B}_{0}$ performing a gyration motion around the large-scale magnetic field lines. The small superposed irregularities interact with the gyrating particles in such a way that the first adiabatic invariant (the magnetic moment) is not conserved, leading to a scattering of the pitch angle, $\theta$ (angle between the velocity vector $\boldsymbol{v}$ of the particles and $\boldsymbol{B}_{0}$ ). As can be seen, for example, in the first chapter of Shalchi (2009), this pitch-angle scattering leads to a diffusive motion of the particle's guiding centers along $\boldsymbol{B}_{0}$.

Whereas early transport models (Meyer et al. 1956) are based on a simple, empirically found, one-dimensional diffusion equation in space, later and more complete models describe the evolution of the phase-space density in the test-particle approach

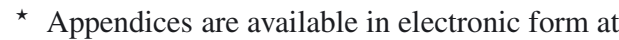
http: //wwW . aanda.org by a Fokker-Planck type transport equation that can be derived from the equation of continuity in phase space. This equation may take additional effects into account that arise, for example, from the spatial variation in the guiding magnetic field. A lot of work has been done in simulating this transport by numerically solving Fokker-Planck equations that include different effects, such as adiabatic focusing, adiabatic cooling or perpendicular diffusion, assuming different types of magnetic turbulence that lead to different shapes of the diffusion coefficients (for review see Dröge \& Kartavykh 2009; Dröge et al. 2010; Dröge 2003; Agueda et al. 2008; Qin et al. 2004; Ruffolo 1995).

For an analytical treatment, the Fokker-Planck type transport equation has to be simplified. This is normally done by assuming a near isotropic distribution function and applying a diffusion approximation to the transport equation. The result is again a diffusion equation, known from the early models describing cosmic ray transport, which can be solved analytically in many cases. The advantage of this circuitous derivation of the diffusion equation is that some of the previously described transport effects can be accounted for in the diffusion regime. In this work we concentrate on the effect of adiabatic focusing. 
Adiabatic focusing arises in the case of a slowly varying guide magnetic field $\boldsymbol{B}_{0}$. "Slowly" means that the typical length scale that characterizes the change in $B_{0}$ is large compared to the spatial dimension of the particles' gyration motion. In this case it can be assumed that $\boldsymbol{B}_{0}$ is constant for a single gyration, and the first adiabatic invariant is conserved. For a particle moving in a diverging magnetic field, this conservation of the magnetic moment leads to an energy transfer from the perpendicular to the parallel motion, resulting in a decreasing pitch angle, which justifies the name "adiabatic focusing". For a particle moving in a converging magnetic field, the energy transfer is vice versa. The total energy is conserved in both scenarios.

In Sect. 2 of this paper we present an analytical solution to the one-dimensional focused diffusion equation, where we assume a spatial power-law dependence for the spatial diffusion coefficient $\kappa$ and the background magnetic field $\boldsymbol{B}_{0}$. In Sects. 3 and 4 we derive the corresponding intensity- and anisotropytime profiles. We compare the analytical results with in-situ measurements of solar electrons in Sect. 5. We summarize this work in Sect. 6.

\section{The focused diffusion equation}

The time-dependent one-dimensional diffusion equation including the effect of adiabatic focusing reads as (Earl 1976) ${ }^{1}$

$\frac{\partial F}{\partial t}-\frac{\partial}{\partial z}\left(\kappa\left[\frac{\partial F}{\partial z}-\frac{F}{L}\right]\right)=S(z, p, t)$,

where

$F(z, p, t)=\frac{1}{2} \int_{-1}^{1} f(z, p, \mu, t) \mathrm{d} \mu$

denotes the isotropic part of the assumed gyrotropic phase-space density $f(z, p, \mu, t), \mu$ is the pitch-angle cosine, $L$ is the focusing length,

$\frac{1}{L}=-\frac{1}{B_{0}(z)} \frac{\partial B_{0}(z)}{\partial z}$

and $S(z, p, t)$ represents the source distribution.

Assuming a point-like injection source in time and space

$S(z, p, t)=S_{1}(p) \delta\left(z-z_{0}\right) \delta\left(t-t_{0}\right)$,

and a spatial power-law dependence for the guide magnetic field $B_{0}(z)$ and the diffusion coefficient $\kappa(z)$,

$$
\begin{aligned}
B_{0}(z) & \propto\left(\frac{z+z_{1}}{z_{2}}\right)^{m}, \\
\kappa(z) & =\kappa_{0}(p)\left(\frac{z+z_{1}}{z_{2}}\right)^{\alpha},
\end{aligned}
$$

the diffusion Eq. (1) reads as ${ }^{2}$

$\frac{\partial F}{\partial T}-\frac{1}{z_{2}^{2}} \frac{\partial}{\partial \tilde{z}}\left(\kappa_{0} \tilde{z}^{\alpha}\left[\frac{\partial F}{\partial \tilde{z}}+\frac{m}{\tilde{z}} F\right]\right)=\frac{S_{1}(p)}{z_{2}} \delta\left(\tilde{z}-\tilde{z}_{0}\right) \delta(T)$

\footnotetext{
1 The equation presented in Earl (1976) looks slightly different because of a different definition of the phase-space density: $F_{\text {Earl }}=F B_{0}$.

2 Though it is not the same mathematical function, we use the notation $F(\tilde{z}, T)=F(z(\tilde{z}), t(T))$ throughout this paper to describe the same physical quantity.
}

with the abbreviations,

$\tilde{z}(z):=\frac{z+z_{1}}{z_{2}}, \quad \tilde{z}_{0}:=\frac{z_{0}+z_{1}}{z_{2}}, \quad T:=t-t_{0}$.

Here $z_{1} \geq 0$ denotes a translation to avoid an infinitely large magnetic field at the Sun $(z=0)$ for $m<0$, and $z_{2}>0$ denotes a scaling length to introduce dimensionless quantities.

Furthermore, we defined the particles mean free path

$\lambda(z):=\frac{3 \kappa(z)}{v}$,

with the particles velocity $v$, according to the mean free path of a three-dimensional self-diffusion process ${ }^{3}$.

For the boundary conditions mentioned below, Eq. (7) can be solved analytically. The solution for $\alpha \neq 2$ is given by

$$
\begin{aligned}
F(\tilde{z}, p, T)= & \frac{3 S_{1} z_{2} \tilde{z}_{0}^{m}\left(\tilde{z} \tilde{z}_{0}\right)^{\frac{1-m-\alpha}{2}}}{|2-\alpha| \lambda_{0} v T} \exp \left(-3 z_{2}^{2} \frac{\tilde{z}^{2-\alpha}+\tilde{z}_{0}^{2-\alpha}}{(2-\alpha)^{2} \lambda_{0} v T}\right) \\
& \times I_{\left|\frac{\alpha-m-1}{2-\alpha}\right|}\left(\frac{6 z_{2}^{2}\left(\tilde{z} \tilde{z}_{0}\right)^{\frac{2-\alpha}{2}}}{(2-\alpha)^{2} \lambda_{0} v T}\right),
\end{aligned}
$$

where $I_{|\xi|}$ represents the modified Bessel function of the first kind and $\lambda_{0}=\frac{3 \kappa_{0}}{v}$ the mean free path at $z=z_{2}-z_{1}$ (see Appendix A for details). In the case of a linear spatial dependence of the mean free path $\lambda(\alpha=1)$ and a positive focusing length $L(m<0)$, this solution agrees with the one presented in Kocharov et al. (1996) for $z_{1}=0$. A similar solution in a different context can also be found in Toptygin (1985).

Though the presented solution (10) solves Eq. (7) for $\alpha \in \mathbb{R} \backslash\{2\}$ and $m \in \mathbb{R}$, we reduce our analysis to the case

$\xi:=\frac{\alpha-m-1}{2-a} \geq 0$

where (10) fulfills the following physically meaningfull initial and boundary conditions:

i) $F\left(t=t_{0}\right)$ vanishes everywhere except at the source:

$$
\lim _{t \rightarrow t_{0}} F(t)=0 \quad \text { for } \quad z \neq z_{0}
$$

ii) the particle flux density,

$$
J(z, p, T)=-\kappa\left[\frac{\partial F}{\partial z}-\frac{F}{L}\right],
$$

vanishes for $z \rightarrow \infty$,

$\lim _{z \rightarrow \infty} J(z)=0$,

and is small for $z \rightarrow 0$,

$\lim _{z \rightarrow 0} J(z)=$ finite

for $z_{1} \ll z_{2}$ the boundary condition (15) can be approximated by

$$
\lim _{z \rightarrow 0} J(z) \approx \lim _{\tilde{z} \rightarrow 0} J(\tilde{z})=0 .
$$

3 There is a difference between three-dimensional isotropic selfdiffusion and the diffusion caused by pitch-angle scattering. In the latter case the mean free path is a definition. 
S. Artmann et al.: A diffusive description of the focused transport of solar energetic particles

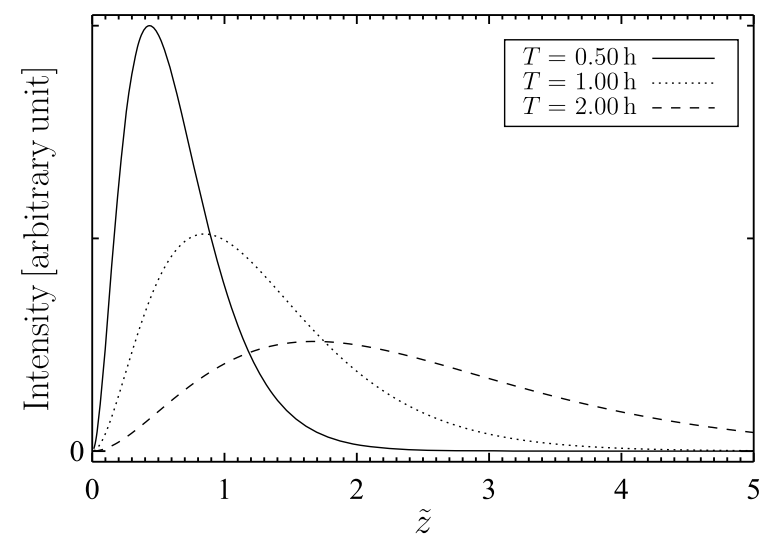

Fig. 1. Sketch of the temporal evolution of the spatial intensity distribution of solar energetic particles released instantaneously at $T=0$ for $\alpha=1$ and $m=-2$.

This means that the number of particles between the Sun $(z=0)$ and infinity $(z=\infty)$ is conserved.

In the case of a constant magnetic field and a constant diffusion coefficient ( $m=\alpha=0$ ) there is no need for the translation $z_{1}$, and we can set $z_{1}=0$. In this case Eq. (10) simplifies to

$F(z, T)=\frac{S_{1}}{\sqrt{\pi \kappa_{0} T}} \exp \left(-\frac{z^{2}+z_{0}^{2}}{4 \kappa_{0} T}\right) \sinh \left(\frac{z z_{0}}{2 \kappa_{0} T}\right)$,

in agreement with the solution of the focused diffusion Eq. (1) for constant $\kappa=\kappa_{0}$, and $L$ that fulfills the boundary conditions $(12,14)$ and $\lim _{z \rightarrow 0} F(z)=0$

$F(z, T)=\frac{S_{1}}{\sqrt{\pi \kappa_{0} T}} \exp \left(-\frac{\left(z-z_{0}-\frac{\kappa_{0} T}{L}\right)^{2}+2 z_{0} z}{4 \kappa_{0} T}\right) \sinh \left(\frac{z z_{0}}{2 \kappa_{0} T}\right)$

in the limiting case $L \rightarrow \infty$.

\section{Intensity profiles}

The omnidirectional intensity $\bar{I}(\tilde{z}, p, T)$ is connected to the isotropic phase-space density by the simple relation (Rossi \& Olbert 1970):

$\bar{I}(\tilde{z}, p, T)=p^{2} F(\tilde{z}, p, T)$.

By inserting Eq. (10) into (19) we get an analytic expression for the omnidirectional intensity depending on the time $T$, the position $z$, the spatial diffusion coefficient $\kappa=\kappa_{0} \tilde{z}^{\alpha}$, and the power-law dependence of the magnetic field $m$.

Figure 1 shows the temporal evolution of the spatial intensity distribution. Energetic particles are released at the Sun instantaneously at $T=0$, represented by a delta-function $\delta(T)$. Due to pitch-angle scattering, this delta function develops into a Gaussian with a time-dependent FWHM. The effect of adiabatic focusing and the assumed boundary conditions lead to a modification of this Gaussian by the Bessel function $I_{\xi}$, which results in an a outward movement of the bulk of the particles. This seems to be a convincing solution, but only to a certain degree. It cannot be avoided that a (Markovian) diffusive description of particle propagation violates the basic causality law of special relativity. For times $T>0$, the asymptotic behavior of the intensity profile $\lim _{z \rightarrow \infty} \bar{I}(z)=0$ includes a nonvanishing probability of particles

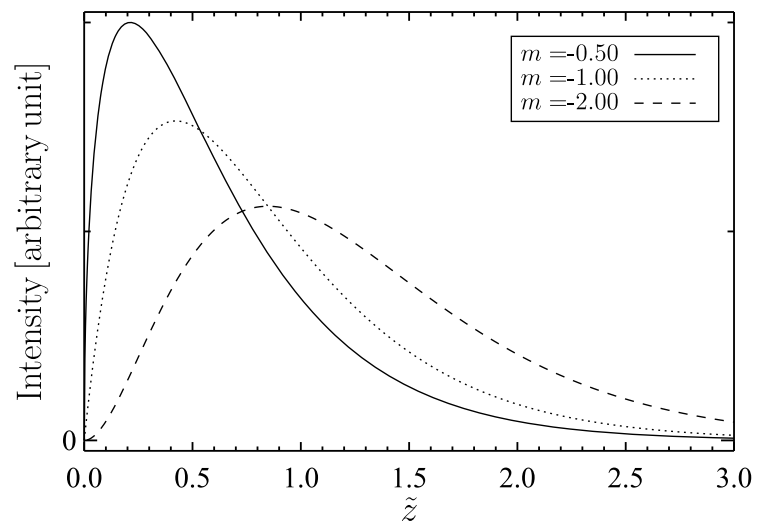

Fig. 2. Sketch of the spatial dependence of the intensity distribution on the magnetic field structure (Eq. (5)) at a given time $T=1 \mathrm{~h}$ for $\alpha=1$.

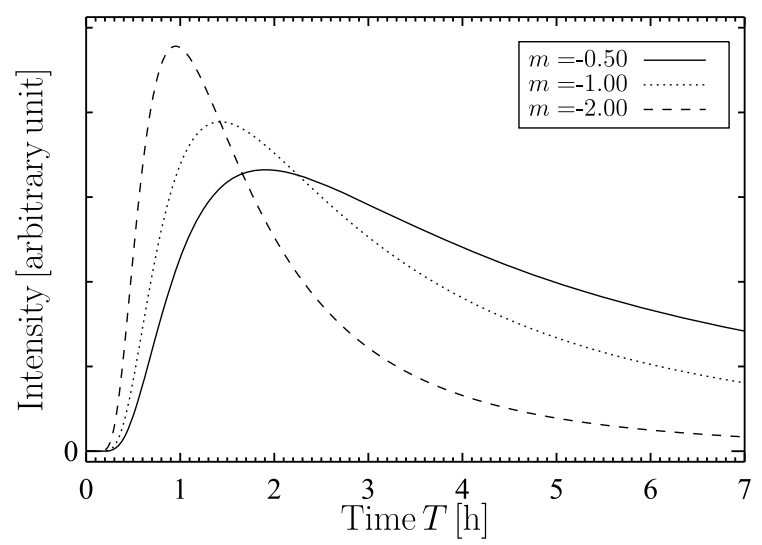

Fig. 3. Sketch of the temporal dependence of the intensity distribution on the magnetic field structure (Eq. (5)) at a given position $\tilde{z}=1.2$ for $\alpha=1$.

travelling faster than the speed of light. A non-Markovian generalization of this diffusion model, as described in Dunkel et al. (2007), could avoid this violation.

Figures 2 and 3 sketch the influence of the magnetic field spatial variation (Eq. (5)) on the intensity-time and intensityspace profile. It can be seen that particles in a diverging magnetic field with $m=-2$ propagate faster in the parallel direction than those in a field with $m=-1$ and with $m=-0.5$. The physical explanation for this is the effect of adiabatic focusing that transfers energy from gyration to translation along the field lines in a diverging field and that has a stronger effect for $m=-2$.

Figures 4 and 5 illustrate the dependence of the omnidirectional intensity on spatial variations of the diffusion coefficient (or mean free path). For better comprehension of the displayed curve progressions Fig. 6 shows the mean free path, $\lambda$, as a function of the distance $\tilde{z}$. Combining Figs. 4 and 6 , we see that a short mean free path results in a slow and a long mean free path in a fast diffusion process. This means that particles spend more time in an area with a short mean free path, as expected. It can also be seen that the area under the curves in Fig. 4 is the same. This is a consequence of our postulated boundary conditions where we required a conservation of the number of particles between $\tilde{z}=0$ and $\tilde{z}=\infty$. Figure 5 exhibits a lower density of particles moving with a higher bulk velocity due to a larger mean free path. 


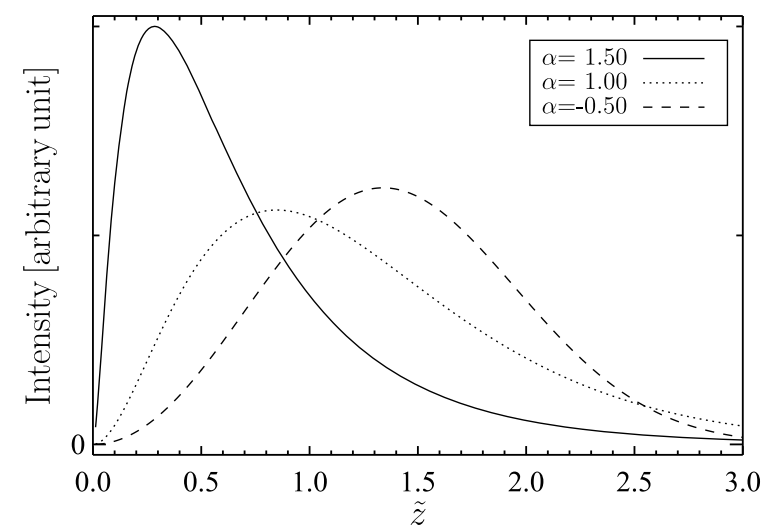

Fig. 4. Sketch of the spatial dependence of the intensity distribution on the spatial power-law dependence of the diffusion coefficient at a given time $T=1 \mathrm{~h}$ for $m=-2$.

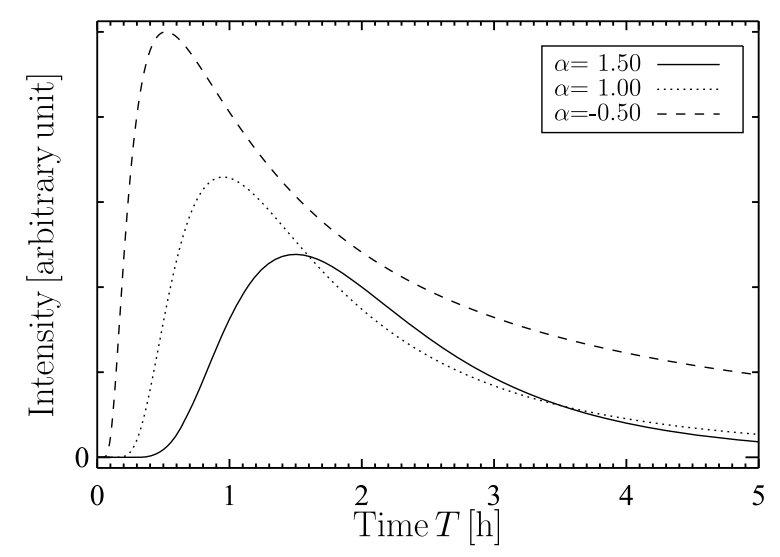

Fig. 5. Sketch of the temporal dependence of the intensity distribution on the spatial power-law dependence of the diffusion coefficient at a given position $\tilde{z}=1.2$ for $m=-2$.

\section{Anisotropy-time profiles}

Beside the omnidirectional intensity, which carries information about the particle flux averaged over the solid angle, the diffusive description also allows for directional information of the particle propagation. This information can be expressed in terms for the anisotropy of the particle distribution. For a gyrotropic phasespace density $f(z, p, \mu, T)$, the first-order anisotropy $A(z, p, T)$ is defined as

$A(z, p, T)=3 \frac{\int_{-1}^{1} f(z, p, \mu, T) \mu \mathrm{d} \mu}{\int_{-1}^{1} f(z, p, \mu, T) \mathrm{d} \mu}$.

Because $f(z, p, \mu, T) \geq 0$ is proportional to the probability distribution, the anisotropy $A$ represents three times the expectation value of the pitch-angle cosine $\mu$ and is limited by

$|A(z, p, T)| \leq 3$

If we express $f(z, p, \mu, T)$ in terms of the isotropic and anisotropic part, $F(z, p, T)$ and $g(z, p, \mu, T)$, of the phase-space density

$f(z, p, \mu, T)=F(z, p, T)+g(z, p, \mu, T)$,

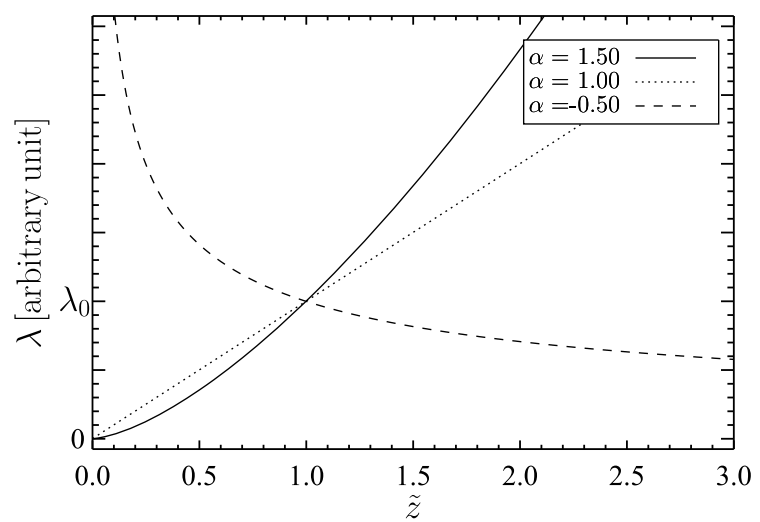

Fig. 6. Sketch of the spatial dependence of the mean free path $\lambda$ for three different powers.

we find

$A(z, p, T)=3 \frac{\int_{-1}^{1} \mu g(z, p, \mu, T) \mathrm{d} \mu}{2 F(z, p, T)}$.

According to the diffusion approximation presented in Schlickeiser et al. (2007) and Schlickeiser \& Shalchi (2008), the anisotropic part, $g(z, p, \mu, T)$, can be expressed in the weak focusing limit and for negligible perpendicular diffusion as

$g(z, p, \mu, T)=\overbrace{\left[\int_{-1}^{1} \frac{(1-\mu) D_{\mu p}(\mu)}{D_{\mu \mu}(\mu)} \mathrm{d} \mu-2 \int_{-1}^{\mu} \frac{D_{\mu p}(x)}{D_{\mu \mu}(x)} \mathrm{d} x\right] \frac{1}{2} \frac{\partial F}{\partial p}}^{=g_{\mathrm{cg}}}$
$+\underbrace{\left[\int_{-1}^{1} \frac{(1-\mu)\left(1-\mu^{2}\right)}{D_{\mu \mu}(\mu)} \mathrm{d} \mu-2 \int_{-1}^{\mu} \frac{\left(1-x^{2}\right)}{D_{\mu \mu}(x)} \mathrm{d} x\right] \frac{v}{4}\left(\frac{\partial F}{\partial z}-\frac{F}{L}\right)}_{=g_{\mathrm{s}}}$.

Evidently, $g(z, p, \mu, T)$ consists of a Compton-Getting part, $g_{\mathrm{cg}}$, and a streaming part, $g_{\mathrm{s}}$. Consequently, we split our anisotropy into two parts,

$A(z, p, T)=A_{\mathrm{s}}(z, p, T)+A_{\mathrm{cg}}(z, p, T)$,

and by inserting Eq. (23) into (22) we find

$$
\begin{aligned}
& A_{\mathrm{s}}(z, p, T)=-3 \frac{\kappa}{v}\left[\frac{\partial \ln (F)}{\partial z}-\frac{1}{L}\right]=-\lambda\left[\frac{\partial \ln \left(F B_{0}\right)}{\partial z}\right] \\
& A_{\mathrm{cg}}(z, p, T)=-\frac{3 a_{11}}{4} \frac{\partial \ln (F)}{\partial p}
\end{aligned}
$$

where we identified the spatial diffusion coefficient,

$\kappa=\frac{v^{2}}{8} \int_{-1}^{1} \frac{\left(1-\mu^{2}\right)^{2}}{D_{\mu \mu}} \mathrm{d} \mu$

and introduced the rate of adiabatic deceleration:

$a_{11}=\int_{-1}^{1} \frac{\left(1-\mu^{2}\right) D_{\mu p}}{D_{\mu \mu}} \mathrm{d} \mu$.

It is important to notice that the diffusion approximation only holds if the phase-space density $f(z, p, \mu, T)$ is close to the 
isotropic equilibrium distribution $F(z, p, T)$, which means $|g(z, p, \mu, T)| \ll F(z, p, T)$. If the anisotropic part exceeds the isotropic one, Eq. (23) no longer accounts for the anisotropic part. A violation of this requirement gives rise to negative values of the phase-space density (21) and causes absolute values of the anisotropy to be higher than three. The anisotropy is therefore an indicator of the validity of the diffusive particle transport description.

As shown in Schlickeiser et al. (2009), the ComptonGetting anisotropy (Eq. (26)) is small compared to the streaming anisotropy (Eq. (25)) for solar energetic particles observed at 1 AU. Therefore it will be neglected.

We use Eqs. (25) and (10) to calculate the anisotropy-time profile of solar energetic particles with the assumptions of a spatial power-law dependence for the magnetic field $B_{0}(z)$ and the diffusion coefficient $\kappa(z)$ and find for $\xi=\frac{\alpha-m-1}{2-\alpha} \geq 0$ :

$A_{\mathrm{S}}=\frac{3 \tilde{z} z_{2}\left[1-\left(\frac{\tilde{z}_{0}}{\tilde{z}}\right)^{\frac{2-\alpha}{2}} \frac{I_{\xi+1}\left(\frac{2 z_{2}^{2}\left(\tilde{z} \tilde{z}_{0}\right)^{\frac{2-\alpha}{2}}}{(2-\alpha)^{2} \kappa_{0} T}\right)}{I_{\xi}\left(\frac{2 z_{2}^{2} 2_{\left(z z_{0}\right)^{2}} \frac{2-\alpha}{2}}{(2-\alpha)^{2} \kappa_{0} T}\right)}\right]}{(2-\alpha) v T}$.

Under typical magnetic field and solar wind conditions, this formal expression for the anisotropy can be simplified in most cases for solar energetic particles observed at $z \approx 1.2 \mathrm{AU}$. For a magnetic field close to the nominal Parker spiral with $m=-2$ and for a particle injection in the corona at three solar radii $z_{0}=0.014 \mathrm{AU}$ with a typical magnetic field strength of $B_{0}(z=$ $\left.z_{0}\right)=30 \mu \mathrm{T}$ and a particle detection close to the Earth with a magnetic field strength of $B_{0}(z=1.2 \mathrm{AU})=5 \mathrm{nT}$, we choose the scaling length $z_{2}=1 \mathrm{AU}$ and have to set the translation to $z_{1}=0.0015 \mathrm{AU}$. This implies for the observed anisotropy-time profile at $z=1.2 \mathrm{AU}: \tilde{z}_{0} \ll \tilde{z}$. Keeping this relation in mind we take a look at the monotonically increasing function

$0 \leq \frac{I_{\xi+1}(x)}{I_{\xi}(x)} \leq 1 \quad \forall \quad x \in \mathbb{R} \quad \wedge \quad \xi \in \mathbb{R}_{0}^{+}$

with

$\lim _{x \rightarrow 0} \frac{I_{\xi+1}(x)}{I_{\xi}(x)}=0 \quad \forall \quad \xi \in \mathbb{R}_{0}^{+}$

$\lim _{x \rightarrow \infty} \frac{I_{\xi+1}(x)}{I_{\xi}(x)}=1 \quad \forall \quad \xi \in \mathbb{R}_{0}^{+}$

and conclude that the second summand in Eq. (29) is small compared to the first if $\alpha$ is small enough compared to 2 . In this case the anisotropy simplifies to

$A_{\mathrm{s}}(z, p, T) \approx \frac{3 z}{(2-\alpha) v T}$ for $\quad \alpha<2$.

Figure 7 shows a sketch of the exact streaming anisotropy (Eq. (29)) in comparison with the approximated anisotropy (Eq. (33)) for three different values of $\alpha$. The sketch confirms that the approximation holds if $\alpha$ is small enough compared to 2. For $\alpha=-0.5$ and $\alpha=1.0$, there is only a negligible deviation, but there is a significant deviation for $\alpha=1.5$. For typical solar wind conditions and a particle observation at $z \approx 1.2 \mathrm{AU}$, it can roughly be said that the approximation holds for $\alpha \leq 1$. In this case the anisotropy is independent of the global magnetic field structure and the absolute value of the mean free path represented by $m$ and $\lambda_{0}$. There is also only a weak dependence for $\alpha>1$. On the other hand, Fig. 7 proves that the anisotropy is sensitive to the spatial variation of the diffusion coefficient $\kappa$.

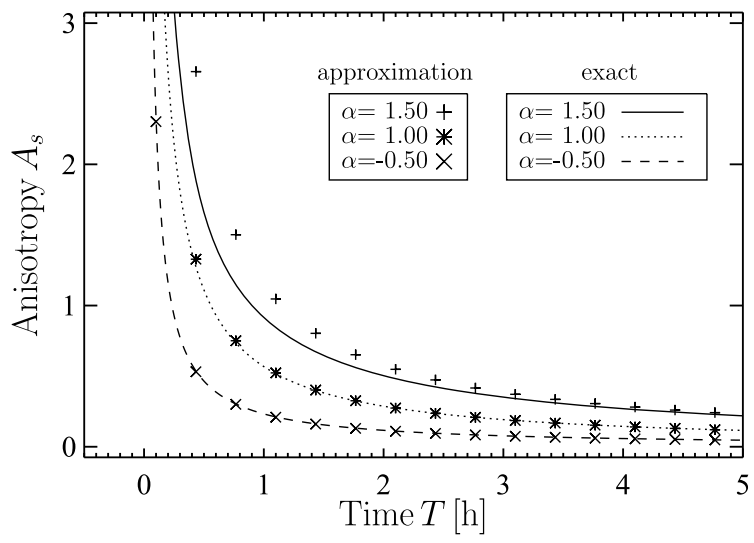

Fig. 7. Sketch of the anisotropy-time profile (Eq. (29)) in comparison with the approximation (Eq. (33)) for three different values for $\alpha$.

It should be mentioned that the anisotropy tends to infinity for $t \rightarrow t_{0}$, in contrast to definition (22) that implies a maximum value of three for the anisotropy. This erratic behavior is a consequence of the violation of the causality law of special relativity discussed in Sect. 3 and the limited scope for applying of the diffusion approximation. The initial particle flux that violates the causality law and reaches the position of detection just after injection is highly anisotropic. The required assumption $|g(z, p, \mu, T)| \ll F(z, p, T)$ of the diffusion approximation is not fulfilled and, as discussed before, anisotropy values over three arise. For later times and lower anisotropy values, the accuracy of the diffusion approximation increases and the anisotropy values become more realistic.

\section{Comparison with measured data}

The expressions derived in the previous sections for the evolution of the intensity (Eqs. (10) and (19)) and the anisotropy (Eq. (22)) can be compared with in situ measurements during solar energetic electron events. We use electron intensities observed by the three-dimensional plasma and energetic particle (3DP) experiment onboard the Wind spacecraft (Lin et al. 1995). The semi-conductor detector telescopes (SST) of Wind/3DP measure electrons in the energy range $27-512 \mathrm{keV}$ with a full $4 \pi$ angular coverage in one spacecraft spin period. The pitchangle distributions provided by SST have a $22.5^{\circ}$ resolution and allow the intensity- and anisotropy-time profiles to be calculated for a given event. An exceptionally large solar particle event occurred on 2001 April 15 in association with an X14.4 solar flare located at $\mathrm{S} 20 \mathrm{~W} 85$, a type III radio burst and a coronal mass ejection. To reduce the very large number of free parameters in Eqs. (10) and (22) we assume a particle injection at approximately three solar radii $\left(z_{0}=0.014 \mathrm{AU}\right)$ and a distance of $z=1.2 \mathrm{AU}$ along the Archimedean spiral from the Sun to the spacecraft. Furthermore, we assume a magnetic field strength of $B_{0}(z=0)=30 \mu \mathrm{T}$ at the Sun.

The MFI instrument onboard the Wind spacecraft measured an interplanetary magnetic field strength close to the Earth of approximately $B_{0}(z=1.2 \mathrm{AU})=3 \mathrm{nT}$. We choose the scaling length $z_{2}=1 \mathrm{AU}$ and use Eq. (5) to determine the translation $z_{1}$. We can now vary the free parameters $\alpha, m, \lambda_{0}=\frac{3 \kappa_{0}}{v}$ and $t_{0}$ to find good agreement between the theoretical and the measured profiles. We always determine the source function $S_{1}(p)$ through normalization of our theoretical intensity profile on the measured maximum value for $108 \mathrm{keV}, 180 \mathrm{keV}, 306 \mathrm{keV}$, and $512 \mathrm{keV}$. 

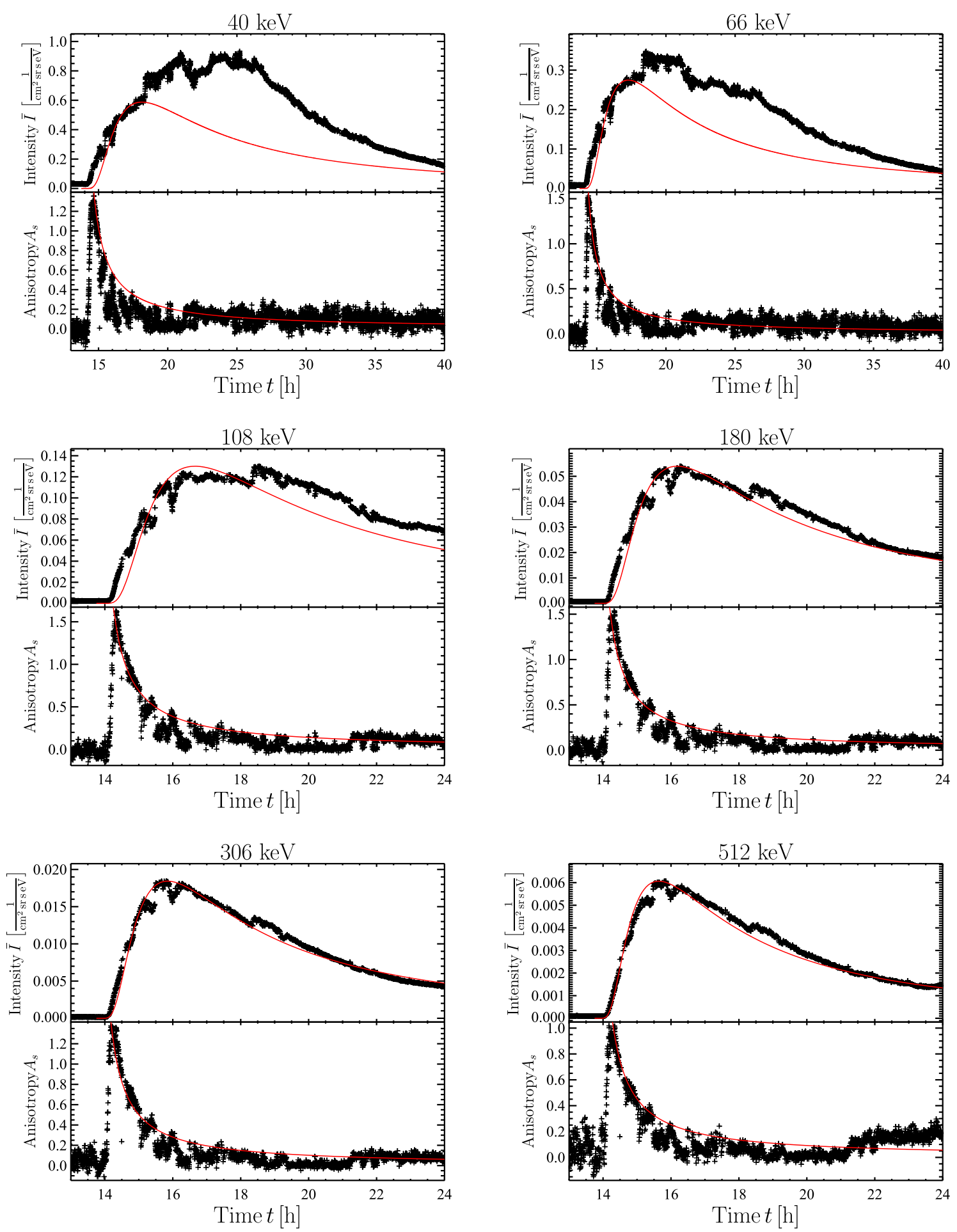

Fig. 8. Anisotropy- and intensity-time profiles for $40-512 \mathrm{keV}$ electrons measured with the Wind/3DP instrument in comparison with the theoretically derived profile (19) with the parametervalues listed in Table 1.

For $27 \mathrm{keV}, 40 \mathrm{keV}$, and $66 \mathrm{keV}$ electrons we use a different value than the maximum value for the normalization. The reason for this choice is the contamination of this profiles with electrons that gained their energy by a different effect than the initial particle injection. This will become more obvious later in this section.

For the set of parameters displayed in Table 1, we plotted the intensity- and anisotropy-time profiles for six different energies in comparison with the measured profiles. As can be seen in Fig. 8 we get a very good fit for electrons with energies
Table 1. Set of parameters that provides a good fit to the data for different energies.

\begin{tabular}{lccc}
\hline \hline$\alpha$ & $m$ & $\lambda_{0}$ & $t_{0}$ \\
\hline 1.0 & -0.74 & $0.173 \mathrm{AU}$ & $13.75 \mathrm{~h}$ \\
\hline
\end{tabular}

of $108 \mathrm{keV}$ and more. For lower energies the intensity fit gets worse. The quality of the anisotropy fit is roughly the same for all six energies. The profiles that are measured in the lowest energy 
channel $(\approx 27 \mathrm{keV})$ reveal poor agreement with the theory and are not shown here. A possible explanation for this growing deviation between measurement and theory for lower electron energies is that particles with lower energies are injected over a longer period of time, which broadens the intensity profile. In addition their injection might be less eruptive, which could explain the slower rise and decay of the measured profile. In this case the assumption of an eruptive injection described by the delta function $\delta\left(t-t_{0}\right)$ no longer holds.

It is remarkable how well this simplified theory agrees with results of much more detailed computer simulations for this event. Bieber et al. (2004) conclude that the onset of particle injection onto the Sun-Earth field line was at 13:42 UT $\pm 1 \mathrm{~min}$, and Agueda et al. (2009) conclude that the electron injection started at 13:48 UT at $80 \mathrm{keV}$. These two previous results are in accordance with our injection time at 13:45 UT. Dröge (2005) modeled the event by assuming a spatially constant radial mean free path for $511 \mathrm{keV}$ electrons and concludes that $\lambda_{r}=0.14 \mathrm{AU}$ $\left(\lambda_{\|}=0.24 \mathrm{AU}\right.$ at Earth). Similarly, Agueda et al. (2009) estimate the radial mean free path of the $62-312 \mathrm{keV}$ electrons is $0.08 \mathrm{AU}\left(\lambda_{\|}=0.17 \mathrm{AU}\right.$ at Earth). At the distance of the Earth these agree with our result:

$\lambda_{\|}(z)=\lambda(z)=0.173\left(z-z_{1}\right) \Rightarrow \lambda(z=1.2 \mathrm{AU}) \approx 0.21 \mathrm{AU}$.

The obtained linear dependence between $\tilde{z}=\frac{z-z_{1}}{z_{2}}$ and the parallel mean free path mainly arises from fitting the anisotropy-time profiles. As already said, the anisotropy depends weakly on the power-law dependence of the magnetic field and the value of the mean free path, $\lambda_{0}$, for the given boundary conditions. At a given position $z$ the slope of the anisotropy-time profile is only determined by the exponent $\alpha$ of the spatial power-law dependence of the diffusion coefficient and the energy of the particles, represented by $v$. We can therefore vary the two parameters $m$ and $\lambda_{0}$ to fit the intensity-time profiles without changing the anisotropy significantly.

Assuming a constant solar wind speed $u_{\mathrm{sw}}$, the model of the Parker spiral (Parker 1958) predicts a radial dependence of the magnetic field strength according to

$B_{0}(r)=B_{0}\left(r_{0}\right)\left(\frac{r_{0}}{r}\right)^{2} \sqrt{1+\left(\frac{\Omega_{\mathrm{S}} r}{u_{\mathrm{sw}}}\right)^{2}}$ for $r>r_{0}$,

where $\Omega_{\mathrm{S}}$ is the sidereal solar rotation rate and $r_{0}$ a radius at which the field is completely frozen into the solar wind. The radial dependence of the pathlength along the Archimedean spiral is given by

$z(r)=\frac{r}{2} \sqrt{1+\left(\frac{\Omega_{\mathrm{S}} r}{u_{\mathrm{sw}}}\right)^{2}}+\frac{u}{2 \Omega_{\mathrm{S}}} \ln \left(\sqrt{1+\left(\frac{\Omega_{\mathrm{S}} r}{u_{\mathrm{sw}}}\right)^{2}}+\frac{\Omega_{\mathrm{S}} r}{u_{\mathrm{sw}}}\right)$.

For $r<u_{\mathrm{sw}} / \Omega_{\mathrm{S}} \approx 1 \mathrm{AU}$ we approximate $z(r) \approx r$ and $B_{0}(r) \approx B_{0}\left(r_{0}\right)\left(\frac{r_{0}}{r}\right)^{2}$ and conclude that $m \approx-2$.

To obtain an acceptable fit we have to deviate from this inverse quadratic dependence of the large scale magnetic field. But the model of the Parker spiral is based on strong simplifications and should be only regarded as an average magnetic field for a longer period of time. For this single event one may also think of particles traveling along a fluxtube with a different spatial dependence of the magnetic field. The deviation may also be caused by the fact that the solar flare location (S20 W85) differs from the footpoint of the Archimedean spiral (S00 W48) assuming the solar wind speed measured at $1 \mathrm{AU}$ of $u_{\mathrm{sw}} \approx 500 \mathrm{~km} \mathrm{~s}^{-1}$.

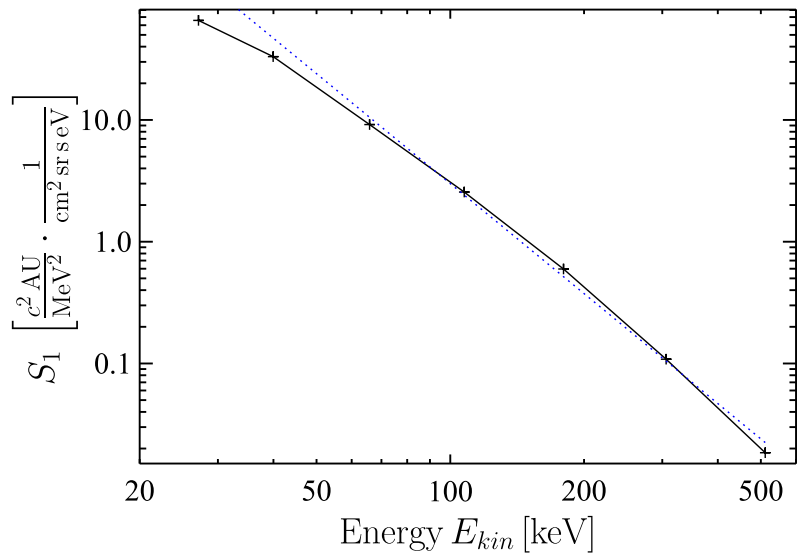

Fig. 9. Energy dependence of the injection function $S_{1}$ (solid black line with crosses) in comparison with a $\propto E_{\text {kin }}^{-3}$ power law (dotted blue line).

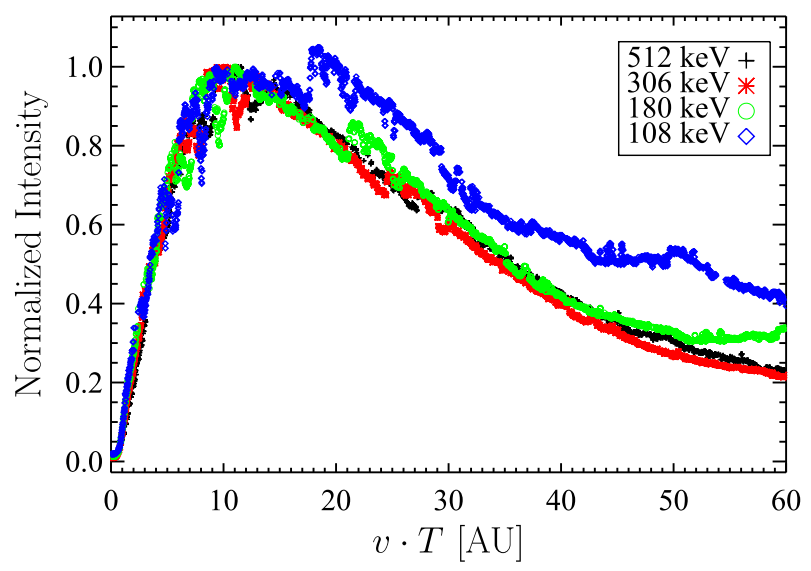

Fig. 10. Normalized intensity-distance profiles for four different electron energies. $v T$ represents the distance that the electrons cover after their first detection at the given position.

Furthermore, we cannot exclude that this different shape of the magnetic field lines is just a local effect in the area where the measurement took place.

Finally, we take a look at the energy dependence of the source function $S_{1}$ that we can infer from the normalization factor. In Fig. 9 we plot $S_{1}\left(E_{\text {kin }}\right)$ against $E_{\text {kin }}$ and notice that $S_{1}$ exhibits a clear proportionality to an $E_{\text {kin }}^{-3}$ power law in the range of the five highest energies. The two lowest energies deviate from this power law and suggest that an exponential decay may also be possible. Considering that the lower energy intensity profiles are strongly contaminated by energetic particles of a different source than the initial injection, one should not overinterpret this deviation. This energy spectrum confirms the result of Heristchi \& Amari (1992) that at least some solar flare electron spectra fit well with a power law in energy in a wide energy range.

Furthermore, our results support the theory of "velocitydependent diffusion" noted for the first time by Bryant et al. (1964). They have shown that the intensity profiles for various energies reveal the same shape if they are plotted against the traveled distance of the particles ( $v T$, where $T$ is the time after the first particles are detected. In our case, $T=t-14 \mathrm{~h}$ ). Figure 10 shows a plot like this for the four highest energy channels of the SST experiment. It can be seen that the variation of all intensity profiles in this energy range is the same and that they only differ in their maximum intensity. 


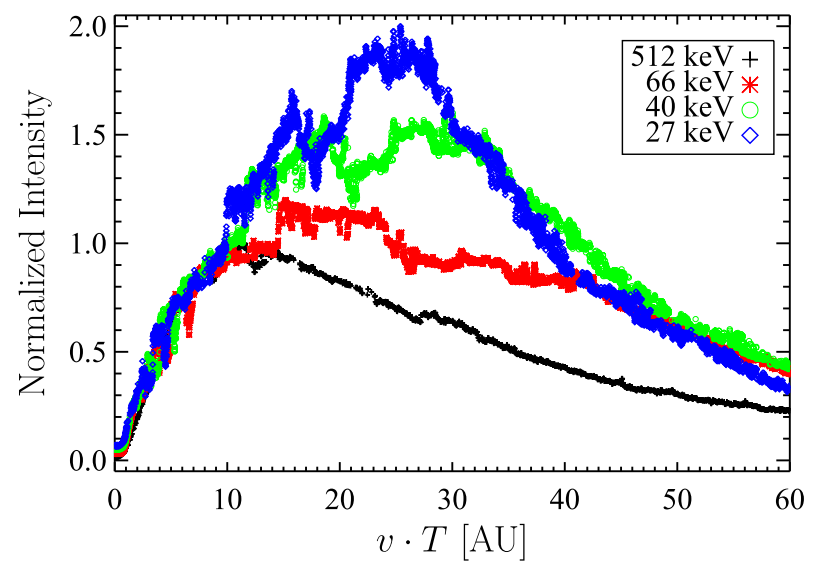

Fig. 11. Normalized intensity-distance profiles for the three lowest energies of Wind/3DP in comparison with the $511 \mathrm{keV}$ electrons.

The sudden deviation that occurs in every profile, with a growing significance for lower energies, is probably caused by an additional injection of mainly lower energetic particles at the Sun. One may also think of an additional acceleration of lower energetic particles at the shock front of the coronal mass ejection that corresponds to this event. Figure 11 demonstrates that this additional disturbance dominates the intensity profiles of the three lowest energies of the SST-F instrument after the rise phase of the intensity profile. This disturbance also explains the poor fit of the intensity-time profiles for $40 \mathrm{keV}$ and $66 \mathrm{keV}$ electrons displayed in Fig. 8 and justifies our normalization to a different value than the measured maximum value for $27 \mathrm{keV}, 40 \mathrm{keV}$, and $66 \mathrm{keV}$ electrons.

Encouraged by our results of energy-independent $\lambda_{0}$ and $\alpha$, we propose the concept of "velocity-dependent diffusion" to the anisotropy. Equations (29) and (33) predict that the anisotropy depends only on $v T$ at a given position for constant $\lambda_{0}$ and $\alpha$. Accordingly, we should get the same profile for each energy if we plot the anisotropy against $v T$. In fact, we discover this feature in the data as can be seen in Fig. 12. This explains our good fits between the measured and the theoretically derived anisotropy for each energy channel with the same set of parameters.

\section{Summary and conclusions}

We solved the one-dimensional diffusion transport equation of solar energetic particles including the effect of adiabatic focusing for a spatial power-law dependence of the large-scale magnetic field $B_{0}$ and the diffusion coefficient $\kappa$. As boundary conditions we assumed a vanishing phase-space density for $t \rightarrow t_{0}$ and a vanishing particle flux at $z \approx 0$ and $z=\infty$. We used our solution to deduce intensity and anisotropy-time profiles that provide a powerful diagnostic tool for estimating the interplanetary particle transport conditions.

We discussed the scope of applying our approximation and pointed out that the anisotropy value is one indicator for the accuracy of our theory. The diffusion approximation does not hold for high anisotropy values. In addition we have to accept that every Markovian diffusion process violates the causality law of special relativity. For a solar flare event, these two sources of error are only influential for a short period of time after the particle injection.

We compared our theoretically derived profiles to the solar 27-512 keV electron event observed by Wind/3DP

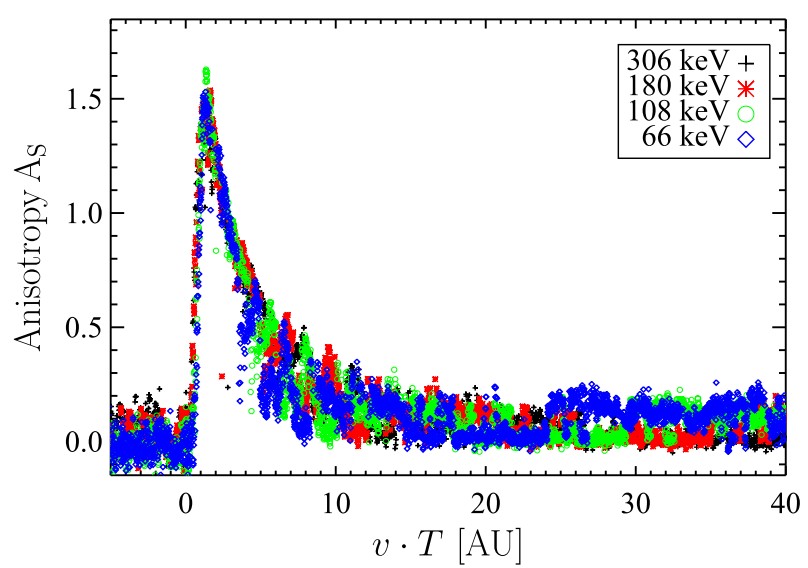

Fig. 12. Anisotropy-distance profiles for four different electron energies.

on 2001 April 15. We find good agreement for electrons with energies of $180 \mathrm{keV}$ up to $512 \mathrm{keV}$. The agreement diminishes for electrons with energies below $180 \mathrm{keV}$, for which an additional acceleration mechanism seems plausible. Beside this, the area of validity is restricted to particles with velocities that are much faster than the solar wind. By fitting the data we find an energyindependent mean free path of approximately $\lambda(z)=0.17 z$ and a spatial dependence of the magnetic field strength given by

$B(z) \propto\left(\frac{z-4.7 \times 10^{-6} \mathrm{AU}}{\mathrm{AU}}\right)^{-0.74}$

In the case of an Archimedean spiral, we would expect an exponent of $m=-2$. We listed several possible explanations for this deviation.

By fitting the data with our theory, we determined the source spectrum of the solar flare electrons and found a $\propto E_{\text {kin }}^{-3}$ power law for energies $E_{\text {kin }} \in \sim[50,500] \mathrm{keV}$. Finally, we confirmed that, in the given energy range, the shape of all intensity profiles may be reduced to one characteristic profile by plotting the data against the "traveled distance since the first particle detection" instead of the time. We proved that this feature of a pure $v T$ dependence is reflected by the anisotropy, too, and is predicted by our theory in the case of energy-independent $\lambda_{0}, m$, and $\alpha$.

We conclude that the transport of solar energetic particles is, at least for some events, a spatial diffusion process that is basically described well by a modified diffusion equation (with a spatial and velocity dependent diffusion coefficient $\kappa \propto v z$ ) that takes the large-scale magnetic field structure into account. Momentum diffusion can be neglected for these timescales.

Acknowledgements. Partial support by the Deutsche Forschungsgemeinschaft through grant Schl 201/23-1 is gratefully acknowledged.

The work at the University of California, Berkeley, was supported by NASA under contracts NNX08AE34G and NAS5-98033.

The Wind 3D Plasma \& Energetic Particle investigation at Berkeley was funded by NASA Grant NNX10AQ31G.

R. P. Lin was also supported in part by the WCU grant (No. R31-10016) funded by the Korean Ministry of Education, Science, and Technology.

\section{References}

Agueda, N., Vainio, R., Lario, D., \& Sanahuja, B. 2008, ApJ, 675, 1601 Agueda, N., Lario, D., Vainio, R., et al. 2009, A\&A, 507, 981 Bieber, J. W., Evenson, P., Dröge, W., et al. 2004, ApJ, 601, L103 Bryant, D. A., Cline, T. L., Desai, U. D., \& McDonald, F. B. 1964, NASA Spec. Publ., 50, 289 
S. Artmann et al.: A diffusive description of the focused transport of solar energetic particles

Dröge, W. 2003, ApJ, 589, 1027

Dröge, W. 2005, Adv. Space Res., 35, 532

Dröge, W., \& Kartavykh, Y. Y. 2009, ApJ, 693, 69

Dröge, W., Kartavykh, Y. Y., Klecker, B., \& Kovaltsov, G. A. 2010, ApJ, 709, 912

Dunkel, J., Talkner, P., \& Hänggi, P. 2007, Phys. Rev. D, 75, 043001

Earl, J. A. 1976, ApJ, 205, 900

Erdelyi, A. 1954, Tables of Integral Transforms (New York: McGraw-Hill), 1 Heristchi, D., \& Amari, T. 1992, Sol. Phys., 142, 209

Kocharov, L. G., Torsti, J., Vainio, R., \& Kovaltsov, G. A. 1996, Sol. Phys., 165, 205

Lin, R. P., Anderson, K. A., Ashford, S., et al. 1995, Space Sci. Rev., 71, 125

Meyer, P., Parker, E. N., \& Simpson, J. A. 1956, Phys. Rev., 104, 768
Parker, E. N. 1958, ApJ, 128, 664

Qin, G., Zhang, M., Dwyer, J. R., \& Rassoul, H. K. 2004, ApJ, 609, 1076

Rossi, B., \& Olbert, S. 1970, Introduction to the physics of space (New York: McGraw-Hill)

Ruffolo, D. 1995, ApJ, 442, 861

Schlickeiser, R., \& Shalchi, A. 2008, ApJ, 686, 292

Schlickeiser, R., Dohle, U., Tautz, R. C., \& Shalchi, A. 2007, ApJ, 661, 185

Schlickeiser, R., Artmann, S., \& Dröge, W. 2009, The Open Plasma Physics Journal, 2,1

Shalchi, A. 2009, Nonlinear Cosmic Ray Diffusion Theories (Berlin: SpringerVerlag)

Toptygin, I. N. 1985, Cosmic rays in interplanetary magnetic fields (Dordrecht: D. Reidel Publishing Company) 


\section{Appendix A: solution of the focused diffusion equation for $\alpha \neq 2$}

Equation (7) can be solved by performing a Laplace transformation on the equation and transferring the corresponding homogeneous second-order differential equation into a modified Bessel differential equation. A particular solution to the inhomogeneous equation can be found with the variation-of-constants method, and the inverse Laplace transformation finally reveals the desired solution.

\section{A.1. Laplace transformation}

Performing a Laplace transformation in the time coordinate to the diffusion Eq. (7) yields, with the boundary condition $\lim _{T \rightarrow 0} F(T)=0$ and after some simplifications,

$$
\frac{\partial^{2} G}{\partial \tilde{z}^{2}}+\frac{\alpha+m}{\tilde{z}} \frac{\partial G}{\partial \tilde{z}}+\left(\frac{m(\alpha-1)}{\tilde{z}^{2}}-\frac{s z_{2}^{2}}{\kappa_{0} \tilde{z}^{\alpha}}\right) G=\frac{-S_{1} z_{2} \delta\left(\tilde{z}-\tilde{z}_{0}\right)}{\kappa_{0} \tilde{z}^{\alpha}}
$$

where

$G(s)=\mathcal{L}\{F(T)\}=\int_{0}^{\infty} F(T) \mathrm{e}^{-s T} \mathrm{~d} T$

is the Laplace transform of $F(T)$.

\section{A.2. Homogeneous solution for $\alpha \neq 2$}

With the substitution

$$
\begin{aligned}
\tilde{z}(\chi) & =\left(\frac{|2-\alpha|}{2 z_{2}} \sqrt{\frac{\kappa_{0}}{s}} \chi\right)^{\frac{2}{2-\alpha}}, \quad \alpha \neq 2 \\
\Leftrightarrow \chi(\tilde{z}) & =\frac{2 z_{2}}{|2-\alpha|} \sqrt{\frac{s}{\kappa_{0}}} \tilde{z}^{\frac{2-\alpha}{2}}
\end{aligned}
$$

and

$$
G(\tilde{z})=\tilde{z}^{\frac{1-\alpha-m}{2}} \mathcal{G}(\chi(\tilde{z})),
$$

Eq. (A.1) transforms into

$$
\begin{aligned}
& \chi^{2} \frac{\partial^{2} \mathcal{G}}{\partial \chi^{2}}+\chi \frac{\partial \mathcal{G}}{\partial \chi}-\left[\chi^{2}+\left(\frac{\alpha-m-1}{2-\alpha}\right)^{2}\right] \mathcal{G}= \\
& -\left(\frac{2}{2-\alpha}\right)^{2} \frac{S_{1} z_{2}}{\kappa_{0}} \delta\left(\tilde{z}-\tilde{z}_{0}\right)\left(\frac{|2-\alpha|}{2 z_{2}} \sqrt{\frac{\kappa_{0}}{s}} \chi\right)^{\frac{3-\alpha+m}{2-\alpha}} .
\end{aligned}
$$

The corresponding homogeneous equation

$\chi^{2} \frac{\partial^{2} \mathcal{H}}{\partial \chi^{2}}+\chi \frac{\partial \mathcal{H}}{\partial \chi}-\left[\chi^{2}+\left(\frac{\alpha-m-1}{2-\alpha}\right)^{2}\right] \mathcal{H}=0$

is the well known modified Bessel differential equation with the two linearly independent solutions:

$\mathcal{H}_{1}(\chi)=I_{|\xi|}(\chi)$

$\mathcal{H}_{2}(\chi)=K_{|\xi|}(\chi)$

where

$\xi=\frac{\alpha-m-1}{2-\alpha}$
Accordingly,

$H_{1}(\tilde{z})=\tilde{z}^{\frac{1-\alpha-m}{2}} I_{\left|\frac{\alpha-m-1}{2-\alpha}\right|}\left(\frac{2 z_{2}}{|2-\alpha|} \sqrt{\frac{s}{\kappa_{0}}} \tilde{z}^{\frac{2-\alpha}{2}}\right)$ and
$H_{2}(\tilde{z})=\tilde{z}^{\frac{1-\alpha-m}{2}} K_{\left|\frac{\alpha-m-1}{2-\alpha}\right|}\left(\frac{2 z_{2}}{|2-\alpha|} \sqrt{\frac{s}{\kappa_{0}}} \tilde{z}^{\frac{2-\alpha}{2}}\right)$

form a fundamental system for the homogeneous differential equation corresponding to Eq. (A.1):

$\frac{\partial^{2} H}{\partial \tilde{z}^{2}}+\frac{\alpha+m}{\tilde{z}} \frac{\partial H}{\partial \tilde{z}}+\left(\frac{m(\alpha-1)}{\tilde{z}^{2}}-\frac{s z_{2}^{2}}{\kappa_{0} \tilde{z}^{\alpha}}\right) H=0$.

\section{A.3. Particular solution for $\alpha \neq 2$}

A particular solution to the inhomogeneous Eq. (A.1) can be found with the variation-of-constants method. According to this method two functions $u_{1}(\tilde{z})$ and $u_{2}(\tilde{z})$ exist, that solve the linear equation system:

$u_{1}(\tilde{z})\left(\begin{array}{l}H_{1}(\tilde{z}) \\ H_{1}^{\prime}(\tilde{z})\end{array}\right)+u_{2}(\tilde{z})\left(\begin{array}{l}H_{2}(\tilde{z}) \\ H_{2}^{\prime}(\tilde{z})\end{array}\right)=\left(\begin{array}{c}0 \\ -\frac{S_{1} z_{2}}{k_{0} \tilde{z}^{\alpha}} \delta\left(\tilde{z}-\tilde{z}_{0}\right)\end{array}\right)$.

The solutions are

$u_{1}(\tilde{z})=\frac{S_{1} z_{2}}{\kappa_{0} \tilde{z}^{\alpha}} \delta\left(\tilde{z}-\tilde{z}_{0}\right) \frac{1}{w} H_{2}(\tilde{z})$

$u_{2}(z)=-\frac{S_{1} z_{2}}{\kappa_{0} \tilde{z}^{\alpha}} \delta\left(\tilde{z}-\tilde{z}_{0}\right) \frac{1}{w} H_{1}(\tilde{z})$

where $w$ is the Wronskian

$w=H_{1}(\tilde{z}) H_{2}^{\prime}(\tilde{z})-H_{1}^{\prime}(\tilde{z}) H_{2}(\tilde{z})=-\frac{2-\alpha}{2} \tilde{z}^{-m-\alpha}$.

A particular solution is given by

$G_{\mathrm{p}}(\tilde{z})=U_{1}(\tilde{z}) H_{1}(\tilde{z})+U_{2}(\tilde{z}) H_{2}(\tilde{z})$,

where $U_{1}(\tilde{z})$ and $U_{2}(\tilde{z})$ are integrals of $u_{1}(\tilde{z})$ and $u_{2}(\tilde{z})$.

For $\alpha<2$ we choose

$U_{1}^{\alpha<2}(\tilde{z})=\int_{\infty}^{\tilde{z}} u_{1}\left(\tilde{z}^{\prime}\right) \mathrm{d} \tilde{z}^{\prime}$

$U_{2}^{\alpha<2}(\tilde{z})=\int_{0}^{\tilde{z}} u_{2}\left(\tilde{z}^{\prime}\right) \mathrm{d} \tilde{z}^{\prime}$,

and the particular solution reads as

$G_{\mathrm{p}}^{\alpha<2}(\tilde{z})=\frac{2 S_{1} z_{2} \tilde{z}_{0}^{m}}{|2-\alpha| \kappa_{0}}\left\{\begin{array}{l}H_{2}\left(\tilde{z}_{0}\right) H_{1}(\tilde{z}) \text { for } \tilde{z}<\tilde{z}_{0} \\ H_{1}\left(\tilde{z}_{0}\right) H_{2}(\tilde{z}) \text { for } \tilde{z}>\tilde{z}_{0} .\end{array}\right.$

For $\alpha>2$ we choose

$U_{1}^{\alpha>2}(\tilde{z})=\int_{0}^{\tilde{z}} u_{1}\left(\tilde{z}^{\prime}\right) \mathrm{d} \tilde{z}^{\prime}$,

$U_{2}^{\alpha>2}(\tilde{z})=\int_{\infty}^{\tilde{z}} u_{2}\left(\tilde{z}^{\prime}\right) \mathrm{d} \tilde{z}^{\prime}$,

and the particular solution reads as

$G_{\mathrm{p}}^{\alpha>2}(\tilde{z})=\frac{2 S_{1} z_{2} \tilde{z}_{0}^{m}}{|2-\alpha| \kappa_{0}}\left\{\begin{array}{l}H_{1}\left(\tilde{z}_{0}\right) H_{2}(\tilde{z}) \text { for } \tilde{z}<\tilde{z}_{0} \\ H_{2}\left(\tilde{z}_{0}\right) H_{1}(\tilde{z}) \text { for } \tilde{z}>\tilde{z}_{0} .\end{array}\right.$

A92, page 10 of 11 


\section{A.4. Inverse Laplace transformation}

The general solution to the differential Eq. (A.1) is the superposition of the homogeneous solutions $H_{1}(\tilde{z})$ and $H_{2}(\tilde{z})$, plus the particular solution $G_{\mathrm{p}}(\tilde{z})$ :

$$
G(\tilde{z}, s)=c_{1}(s) H_{1}(\tilde{z}, s)+c_{2}(s) H_{2}(\tilde{z}, s)+G_{\mathrm{p}}(\tilde{z}, s) .
$$

The constants $c_{1}$ and $c_{2}$ can be determined by the chosen spatial boundary conditions. We cannot find an inverse Laplace transformation for the exponentially growing function $H_{1}(\tilde{z}, s)$ and choose $c_{1}(s)=0$. Using the relation (5.16.42) from Erdelyi (1954) for $a>0$,

$\mathcal{L}^{-1}\left\{\sqrt{s}^{\gamma+2 n} K_{v}(2 \sqrt{a s})\right\}=\frac{(-1)^{n} n ! \sqrt{a}^{v}}{2 T^{n+v+1}} \exp \left(-\frac{a}{T}\right) L_{n}^{v}\left(\frac{a}{T}\right)$,

where $L_{n}^{v}(x)$ denotes the generalized Laguerre polynomials, we find

$F_{n}(\tilde{z}, T)=\tilde{z}^{\frac{1-m-\alpha+(2-\alpha) \mid \xi}{2}} \frac{\exp \left(-\frac{z_{2}^{2} \tilde{z}^{2-\alpha}}{(2-\alpha)^{2} \kappa_{0} T}\right)}{T^{n+|\xi|+1}} L_{n}^{|\xi|}\left(\frac{z_{2}^{2} \tilde{z}^{2-\alpha}}{(2-\alpha)^{2} \kappa_{0} T}\right)$

as homogeneous solutions to the differential Eq. (7). We now perform an inverse Laplace transformation to the particular solution $G_{\mathrm{p}}(\tilde{z}, s)$. Utilizing the relation 5.16.56 from Erdelyi (1954),

$\mathcal{L}^{-1}\left\{K_{v}([\sqrt{a}+\sqrt{b}] \sqrt{s}) I_{v}([\sqrt{a}-\sqrt{b}] \sqrt{s})\right\}=\frac{I_{v}\left(\frac{a-b}{2 T}\right) \mathrm{e}^{-\frac{a+b}{2 T}}}{2 T}$,

where $\mathfrak{R}(a)>0$ and $\mathfrak{R}(b)>0$ has to be fulfilled, we receive

$F_{\mathrm{p}}(\tilde{z}, T)=\mathcal{L}^{-1}\left\{G_{\mathrm{p}}^{\alpha<2}(z, p, s)\right\}=\mathcal{L}^{-1}\left\{G_{\mathrm{p}}^{\alpha>2}(z, p, s)\right\}$

$=\frac{S_{1} z_{2} z_{0}^{m}\left(\tilde{z} \tilde{z}_{0}\right)^{\frac{1-\alpha-m}{2}}}{|2-\alpha| \kappa_{0} T} \exp \left(-z_{2}^{2} \frac{\tilde{z}^{2-\alpha}+\tilde{z}_{0}^{2-\alpha}}{(2-\alpha)^{2} \kappa_{0} T}\right) I_{|\xi|}\left(\frac{2 z_{2}^{2}\left(\tilde{z} \tilde{z}_{0}\right)^{\frac{2-\alpha}{2}}}{(2-\alpha)^{2} \kappa_{0} T}\right)$.

Every superposition

$F(\tilde{z}, T)=F_{\mathrm{p}}(\tilde{z}, T)+\sum_{n} a_{n} F_{n}(\tilde{z}, T)$

is a solution of Eq. (7). A solution fulfilling the boundary conditions (12)-(14) and (16) for $\frac{\alpha-m-1}{2-\alpha} \geq 0$ is given by $F(\tilde{z}, T)=$ $F_{\mathrm{p}}(\tilde{z}, T)$. As an aside, we note that the particular solution is an infinite sum of the homogeneous solutions (A.27):

$F_{\mathrm{p}}=\frac{S_{1} z_{2} \tilde{z}_{0}^{\frac{2-\alpha}{2}(|\xi|-\xi)}}{|2-\alpha| \kappa_{0}}\left(\frac{z_{2}^{2}}{(2-\alpha)^{2} \kappa_{0}}\right)^{|\xi|} \sum_{n=0}^{\infty} \frac{\left(\frac{-z_{2}^{2} \tilde{z}_{0}^{2-\alpha}}{(2-\alpha)^{2} \kappa_{0}}\right)^{n}}{\Gamma(n+|\xi|+1)} F_{n}$.

\section{Appendix B: solution of the focused diffusion equation for $\alpha=2$}

For $\alpha=2$ the Laplace transformed focused diffusion Eq. (A.1) reads as

$\frac{\partial^{2} G}{\partial \tilde{z}^{2}}+\frac{2+m}{\tilde{z}} \frac{\partial G}{\partial \tilde{z}}+\left(m-\frac{s z_{2}^{2}}{\kappa_{0}}\right) \frac{G}{\tilde{z}^{2}}=-\frac{S_{1} z_{2}}{\kappa_{0} \tilde{z}^{2}} \delta\left(\tilde{z}-\tilde{z}_{0}\right)$.

With the ansatz

$H(\tilde{z})=\tilde{z}^{k}$ we find the corresponding homogeneous solutions:

$$
\begin{aligned}
& H_{1}(\tilde{z})=\tilde{z}^{\sqrt{\frac{s z_{2}^{2}}{\kappa_{0}}+\left(\frac{1-m}{2}\right)^{2}}-\frac{m+1}{2}} \\
& H_{2}(\tilde{z})=\tilde{z}^{-\sqrt{\frac{s z_{2}^{2}}{\kappa_{0}}+\left(\frac{1-m}{2}\right)^{2}}-\frac{m+1}{2}} .
\end{aligned}
$$

Again, a particular solution can be found utilizing the variationof-constants method:

$u_{1}(\tilde{z})\left(\begin{array}{l}H_{1}(\tilde{z}) \\ H_{1}^{\prime}(\tilde{z})\end{array}\right)+u_{2}(\tilde{z})\left(\begin{array}{l}H_{2}(\tilde{z}) \\ H_{2}^{\prime}(\tilde{z})\end{array}\right)=\left(\begin{array}{c}0 \\ -\frac{S_{1} z_{2}}{\kappa_{0} \tilde{z}^{2}} \delta\left(\tilde{z}-\tilde{z}_{0}\right)\end{array}\right)$.

The solutions for this linear system of equations are

$u_{1}(\tilde{z})=\frac{S_{1} z_{2}}{\kappa_{0} \tilde{z}^{2}} \delta\left(\tilde{z}-\tilde{z}_{0}\right) \frac{1}{w} H_{2}(\tilde{z})$
$u_{2}(z)=-\frac{S_{1} z_{2}}{\kappa_{0} \tilde{z}^{2}} \delta\left(\tilde{z}-\tilde{z}_{0}\right) \frac{1}{w} H_{1}(\tilde{z})$

with the Wronskian:

$w=H_{1}(\tilde{z}) H_{2}^{\prime}(\tilde{z})-H_{1}^{\prime}(\tilde{z}) H_{2}(\tilde{z})=-2 \tilde{z}^{-m-2} \sqrt{\frac{s z_{2}^{2}}{\kappa_{0}}+\left(\frac{1-m}{2}\right)^{2}}$.

A particular solution for Eq. (B.1) is therefore given by

$G_{\mathrm{p}}(\tilde{z})=U_{1}(\tilde{z}) H_{1}(\tilde{z})+U_{2}(\tilde{z}) H_{2}(\tilde{z})$,

where $U_{1}(\tilde{z})$ and $U_{2}(\tilde{z})$ are integrals of $u_{1}(\tilde{z})$ and $u_{2}(\tilde{z})$.

If we choose

$U_{1}(\tilde{z})=\int_{\infty}^{\tilde{z}} u_{1}\left(\tilde{z}^{\prime}\right) \mathrm{d} \tilde{z}^{\prime}$

$U_{2}(\tilde{z})=\int_{0}^{\tilde{z}} u_{2}\left(\tilde{z}^{\prime}\right) \mathrm{d} \tilde{z}^{\prime}$

the particular solution reads as

$$
\begin{aligned}
G_{\mathrm{p}}(\tilde{z}) & =\frac{S_{1} z_{2}{\tilde{z_{0}}}^{m}}{2 \kappa_{0} \sqrt{\frac{s z_{2}^{2}}{\kappa_{0}}+\left(\frac{1-m}{2}\right)^{2}}}\left\{\begin{array}{l}
H_{2}\left(\tilde{z}_{0}\right) H_{1}(\tilde{z}) \text { for } \tilde{z}<\tilde{z}_{0} \\
H_{1}\left(\tilde{z}_{0}\right) H_{2}(\tilde{z}) \text { for } \tilde{z}>\tilde{z}_{0}
\end{array}\right. \\
& =\frac{S_{1} z_{2} \tilde{z}_{0}^{m}\left(\tilde{z} \tilde{z}_{0}\right)^{-\frac{m+1}{2}}}{2 \kappa_{0} \sqrt{\frac{s z_{2}^{2}}{\kappa_{0}}+\left(\frac{1-m}{2}\right)^{2}}}\left\{\begin{array}{l}
\left(\frac{\tilde{z}}{\tilde{z}_{0}}\right)^{\sqrt{\frac{s z_{2}^{2}}{\kappa_{0}}+\left(\frac{1-m}{2}\right)^{2}}} \\
\left(\frac{\tilde{z}}{\tilde{z}_{0}}\right)^{-\sqrt{\frac{s z_{2}^{2}}{\kappa_{0}}+\left(\frac{1-m}{2}\right)^{2}}} .
\end{array}\right.
\end{aligned}
$$

Using the relation (5.6.6) from Erdelyi (1954)

$\mathcal{L}^{-1}\left\{\frac{1}{\sqrt{s}} \mathrm{e}^{-\sqrt{a} \sqrt{s}}\right\}=\frac{1}{\sqrt{\pi T}} \mathrm{e}^{-\frac{a}{4 T}} \quad$ for $\quad \mathfrak{R}(a) \geq 0$

we find

$F(z, p, t)=\mathcal{L}^{-1}\left\{G_{\mathrm{p}}(z, p, s)\right\}$

$=\frac{S_{1} \tilde{z}_{0}^{m}\left(\tilde{z} \tilde{z}_{0}\right)^{-\frac{m+1}{2}}}{2 \sqrt{\pi \kappa_{0} T}} \exp \left(-\frac{(1-m)^{2} \kappa_{0}^{2} T^{2}+\ln \left(\frac{\tilde{z}}{\tilde{z}_{0}}\right)^{2} z_{2}^{4}}{4 \kappa_{0} T z_{2}^{2}}\right)$

as a solution for the time-dependent focused diffusion equation for $\alpha=2$. 